\title{
A Case of Anterolateral Papillary Muscle Rupture Caused by Isolated First Diagonal Branch Occlusion
}

\author{
Hiroshi Kodama, MD, Shinya Takahashi, MD, PhD, Keijiro Katayama, MD, PhD, Taijiro Sueda, MD, PhD \\ Department of Cardiovascular Surgery, Hiroshima University Hospital, Hiroshima, Japan
}

\section{ABSTRACT}

A 74-year-old man hospitalized due to acute myocardial infarction (AMI) of the first diagonal branch developed cardiogenic shock. Ultrasonography showed anterolateral papillary muscle rupture (PMR) which caused anterior mitral leaflet prolapse and severe mitral valve regurgitation, and he successfully underwent mitral valve replacement. Anterolateral PMR causing anterior mitral leaflet prolapse due to obstruction of the first diagonal branch is rare and should be considered in such an AMI case.

\section{INTRODUCTION}

Anterolateral papillary muscle rupture (PMR) after myocardial infarction is a rare complication caused by disease in multiple coronary arteries. It results in immediate hemodynamic instability and requires surgical mitral valve repair. In the present case, despite the fact that the patient had occlusion of only the first diagonal branch and percutaneous coronary intervention restored flow to the diagonal branch, hemodynamic instability led to a shock state due to partial anterolateral PMR, resulting in prolapse of the anterior leaflet and severe mitral valve regurgitation.

\section{CASE REPORT}

A 74-year-old man consulted our hospital because of chest discomfort. A 12-lead electrocardiogram showed ST-segment elevation in leads I and aVL, and a troponin-T test was strongly positive. Echocardiography showed akinesis of anterolateral left ventricular wall motion and moderate mitral valve regurgitation with mitral valve tethering, and the papillary muscle was not ruptured. Coronary angiography showed obstruction of the proximal segment of the first diagonal branch, and percutaneous coronary intervention was performed using a drugeluting stent, which resulted in slow flow (TIMI 2) (Figures 1A-1C). Intraaortic balloon pumping (IABP) was initiated to maintain flow in the first diagonal branch.

Received fanuary 9, 2017, received in revised form fuly 7, 2017; accepted August 3, 2017.

Correspondence: Shinya Takahashi, Hiroshima University Hospital, Kasumi 1-23 Minami-ku, Hiroshima, Japan 734-8551; +81-82-257-5215; Fax:+81-82257-5219; (e-mailshiny@mte.biglobe.ne.jp).
The patient's hemodyamics became worse the next day. Despite the maximum dose of intravenous catecholamines and IABP support, his physical state deteriorated, with a blood pressure of $70 / 40 \mathrm{mmHg}$, heart rate of 120 beats $/ \mathrm{min}$, and crackles in both lungs. However, his consciousness remained alert. The chest radiograph showed severe bilateral pulmonary edema. The patient was intubated and mechanical ventilation was started immediately. Transthoracic and transesophageal echocardiography revealed the following: a hyperkinetic left ventricle with an ejection fraction of $62 \%$; a slightly reduced motion of the inferior wall, a ruptured anterolateral papillary muscle; and a severe regurgitation jet flowing along the posterior wall of the left atrium, suggesting rupture of the anterolateral papillary muscle (Figures 1D, 1E).

The patient underwent mitral valve replacement with a biological valve on the same day. The anterolateral papillary muscle was ruptured. The valve leaflets and chordae were almost normal. Revascularization of the diagonal branch by coronary artery bypass grafting was not performed, because it did not affect left ventricular function. The patient was weaned from cardiopulmonary bypass with IABP, and the IABP was removed 3 days later.

After this procedure, he developed renal insufficiency and was treated by renal substitution therapy during the postoperative period. Histological examination revealed infiltration of inflammatory cells and solidification necrosis of the anterolateral papillary muscle stump, and interstitial hemorrhage inside of the papillary muscle and at the edge of the stump (Figure 2).

Three months after the operation, he improved enough to walk without assistance and was transferred to another hospital for recovery rehabilitation.

\section{DISCUSSION}

PMR is an uncommon, critical mechanical complication of acute myocardial infarction (AMI), accounting for approximately $5 \%$ of deaths after AMI. The mortality is approximately $50 \%$ within 24 hours when there is medical treatment only and increases to more than $80 \%$ in one week [Nishimura 1983]. PMR often occurs in the posteromedial papillary muscle and develops at a frequency that is 3-12 times higher than anterolateral PMR [Nishimura 1983; Kishon 1992; Bouma 2013], because the posteromedial papillary muscle has a single blood supply from the right coronary artery or the left circumflex coronary artery [Voci 1995]. In contrast, the anterolateral papillary muscle has a dual blood 

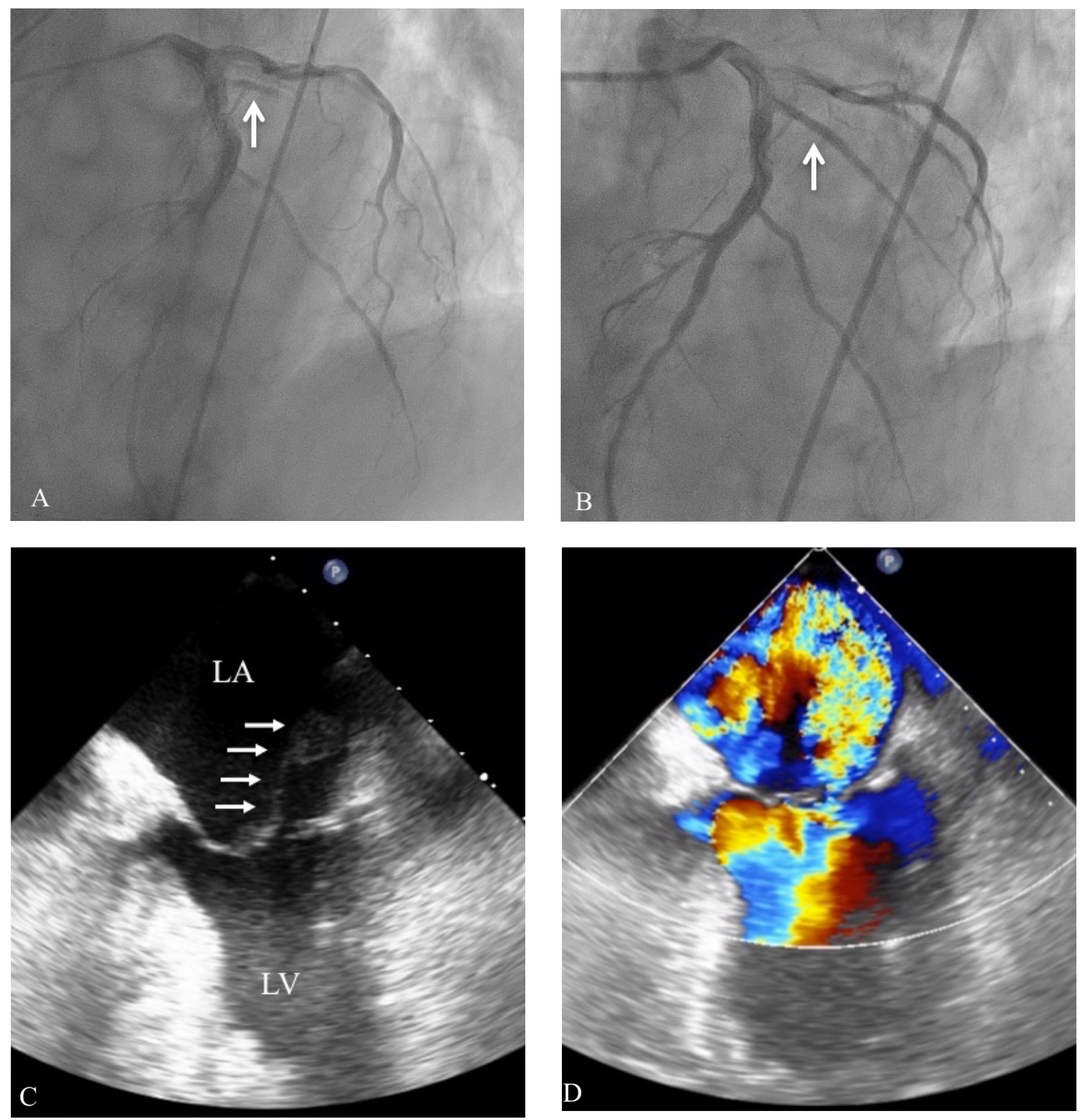

Figure 1, A, Preoperative coronary angiography revealed total occlusion of the first diagonal branch (arrow). B. Percutaneous coronary angioplasty was successfully performed using a drug-eluting stent (arrow). C. Transesophageal echocardiography revealed an echo density in the left atrium (arrow) that suggests a complete ruptured anterolateral papillary muscle. D. Color Doppler echocardiography showed severe mitral regurgitant flow along the posterior wall of the left atrium..

supply in $71 \%$ of patients. Wada et al. reported a similar case and found that the anatomy of a hypoplastic left circumflex branch increased the possibility of PMR by occlusion of only one diagonal branch. This indicates that cardiologists should consider the possibility of a single blood supply for the anterolateral papillary muscle and a risk of its dysfunction or rupture by occlusion even after percutaneous coronary intervention [Wada 2002]. However, in the present case, the left 


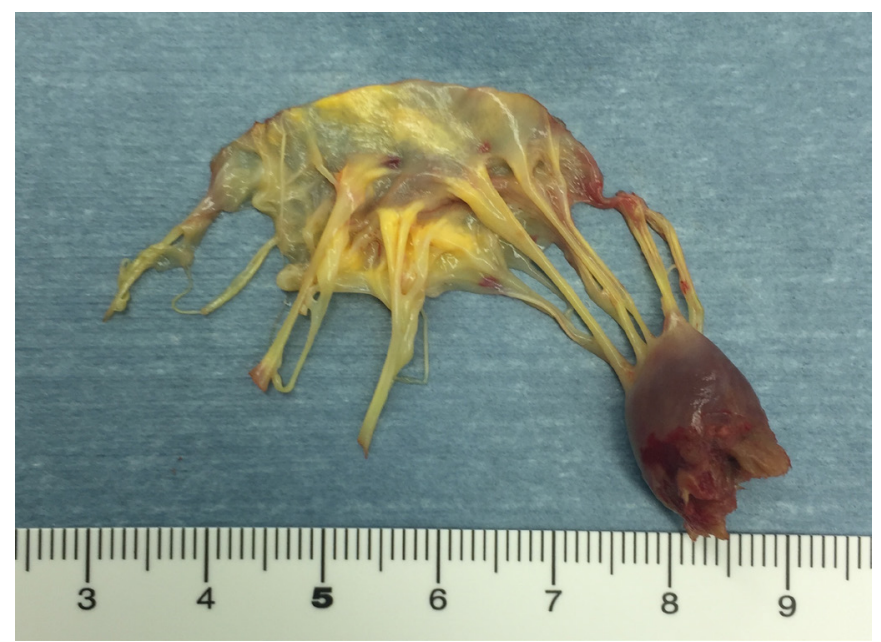

Figure 2. The anterior papillary muscle was completely ruptured at baseline. The papillary muscle showed necrotic degeneration, but the chordae tendinae and mitral leaflet were relatively normal.

circumflex coronary artery was normal. The blood supply was not evaluated in the present case; however, the fact that there was myocardial infarction just below the first diagonal artery on CT may indicate that the blood supply of the infarct area was dominated by the first diagonal branch. The other issue may be that precise diagnosis of blood supply to the papillary muscle has not been clinically established.

The mechanism of PMR is unknown; however, hemodynamic and pathological changes may be related to the occurrence of PMR. Left ventricular ejection fraction is relatively preserved in patients with PMR, which is related to the small area of myocardial infarction [Bouma 2013]. Moreover, the wall motion in the early state of AMI was often hyperkinetic. These changes may cause a greater shearing force between the infarcted papillary muscle and the non-infarcted neighboring myocardium, resulting in PMR. In the present case, because mitral valve regurgitation with tethering remained after coronary artery intervention for diagonal branch, papillary muscle dysfunction might occur and reperfusion led the papillary muscle to rupture.

Pathological findings in PMR often include coagulation necrosis, contraction band necrosis, inflammatory cell infiltration, and interstitial hemorrhage without fibrosis, which reflect the early inflammatory phase of AMI. Interstitial hemorrhage and contraction band necrosis may be related to the dissecting mass of the blood in the papillary muscle and the occurrence of PMR [Fasol 2000].

There are some distinct patterns of PMR. Due to the fact that the anterolateral papillary muscle frequently has a single head and the posteromedial papillary muscle often has several heads, anterolateral PMR is usually complete, whereas posteromedial PMR is often partial. Because complete rupture often causes preoperative death due to hemodynamic instability and cardiogenic shock, complete rupture has a higher mortality than partial or incomplete rupture [Bouma 2014]. The present case was anterolateral and partial PMR, and the patient's deteriorated hemodynamic state was carefully controlled by IABP and catecolamine infusion; therefore, the diagnosis was delayed. This may suggest that a diagnosis of PMR should be considered if there is progressive deterioration of hemodynamics even after treatment of AMI.

Several reports showed that mitral valve repair was suitable in selected cases and has the potential to improve surgical outcome [Fasol 2000; Jouan 2004; Bouma 2013]. However, this case had complete PMR, and repair was not possible because of friable infarcted tissue [Fasol 2000]. Mitral valve replacement may be suitable under the situation of limited experience, unstable hemodynamics, few long-term outcome data and greater technical demands for successful repair.

\section{CONCLUSION}

There are few case reports of anterolateral PMR caused by obstruction of only the first diagonal branch. However, the cardiologist should be aware that obstruction of a diagonal branch has the potential for causing anterolateral PMR, especially in a patient with a large diagonal branch and small left circumflex branch.

\section{REFERENCES}

Bouma W, Wijdh-den Hamer IJ, Klinkenberg TJ, et al. 2013. Mitral valve repair for post-myocardial infarction papillary muscle rupture. Eur J Cardiothorac Surg 44:1063-1069.

Bouma W, Wijdh-den Hamer IJ, Koene BM, et al. 2014. Predictors of inhospital mortality after mitral valve surgery for post-myocardial infarction papillary muscle rupture. J of Cardiothoracic Surg 9:171.

Fasol R, Lakew F, Wetter S. 2000. Mitral repair in patients with a ruptured papillary muscle. Am Heart J 139:549-554.

Jouan J, Tapia M, Cook RC, Lansac E, Acar C. 2004. Ischemic mitral valve pro-lapse: mechanisms and implications for valve repair. Eur J Cardiothorac Surg 26:1112-1117.

Kishon Y, Oh JK, Schaff HV, et al. 1992. Mitral valve operation in postinfarction rupture of a papillary muscle: immediate results and long-term follow-up of 22 patients. Mayo Clin Proc 67:1023-1030.

Nishimura RA, Shaff HV. 1983. Papillary muscle rupture complicating acute myocardial infarction: Analysis of 17 patients. Am J Cardiol $51: 373-377$.

Voci P, Biolotta F, Caretta Q. 1995. Papillary muscle perfusion pattern: A hypothesis for ischemic papillary muscle dysfunction. Circulation 91:1714-1718.

Wada H, et al. 2002. Rupture of the Anterolateral Papillary Muscle Caused by a Single Diagonal Branch Obstruction. Circ J 66:872-873. 